\title{
The spectral density of the scattering matrix of the magnetic Schrödinger operator for high energies
}

\author{
Daniel Bulger and Alexander Pushnitski
}

\begin{abstract}
The scattering matrix of the Schrödinger operator with smooth short-range electric and magnetic potentials is considered. The asymptotic density of the eigenvalues of this scattering matrix in the high energy regime is determined. An explicit formula for this density is given. This formula involves only the magnetic vector-potential.
\end{abstract}

Mathematics Subject Classification (2010). Primary 81U20; Secondary 35P25.

Keywords. Scattering matrix, scattering phase, Schrödinger operator, magnetic field, spectral density.

\section{Main result and discussion}

1.1. Main result. Consider the Schrödinger operator

$$
H=(i \nabla+A)^{2}+V \quad \text { in } L^{2}\left(\mathbb{R}^{d}\right), d \geq 2 ;
$$

here $V: \mathbb{R}^{d} \rightarrow \mathbb{R}$ is an electric potential and $A=\left(A_{1}, \ldots, A_{d}\right): \mathbb{R}^{d} \rightarrow \mathbb{R}^{d}$ is a magnetic vector-potential. We assume that both $V$ and $A$ are infinitely differentiable and satisfy the estimates

$$
\left|\partial^{\alpha} V(x)\right| \leq C_{\alpha}\langle x\rangle^{-\rho-|\alpha|}, \quad\left|\partial^{\alpha} A(x)\right| \leq C_{\alpha}\langle x\rangle^{-\rho-|\alpha|}, \quad \rho>1,
$$

for all multi-indices $\alpha$; here $\langle x\rangle=\left(1+|x|^{2}\right)^{1 / 2}$. Let $H_{0}=-\Delta$; we denote by $S(k)$ the scattering matrix associated with the pair $H, H_{0}$ at the energy $\lambda=k^{2}>0$. We recall the precise definition of the scattering matrix in Section 2.1; here we only note that $S(k)$ is a unitary operator in $L^{2}\left(\mathbb{S}^{d-1}\right)$ and the operator $S(k)-I$ is compact (see e.g. [21] and [20]). Thus, the spectrum of $S(k)$ consists of eigenvalues on the

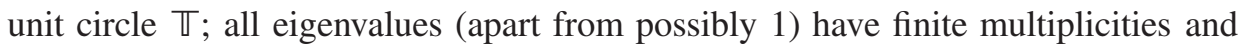
can accumulate only to 1 . Our aim is to describe the asymptotic behavior of these eigenvalues as $k \rightarrow \infty$.

If $A \equiv 0$, one has

$$
\|S(k)-I\|=O\left(k^{-1}\right), \quad k \rightarrow \infty \quad(A \equiv 0),
$$


and so the eigenvalues of $S(k)$ are located on an arc near 1 of length $O\left(k^{-1}\right)$. The large energy asymptotics of $S(k)$ in this case is given by the Born approximation, see e.g. [21], Chapter 8; this makes the analysis of $S(k)$ rather explicit. In [6], using the Born approximation, we have determined the large energy asymptotic density of the spectrum of $S(k)$ for $A \equiv 0$; we will say more about this in the next subsection.

When $A \not \equiv 0$, the situation is radically different: as $k \rightarrow \infty$, the norm $\|S(k)-I\|$ does not tend to zero and the Born approximation is no longer valid. The high energy asymptotic expansion of the scattering amplitude (= the integral kernel of $S(k)-I$ ) for the magnetic Schrödinger operator was obtained (through a very difficult proof) in [19]. Our main result below is a spectral consequence of [19].

We need some notation. For any $\omega \in \mathbb{S}^{d-1}$, let $\Lambda_{\omega} \subset \mathbb{R}^{d}$ be the hyperplane passing through the origin and orthogonal to $\omega$ :

$$
\Lambda_{\omega}=\left\{x \in \mathbb{R}^{d}:\langle x, \omega\rangle=0\right\} .
$$

We equip both $\mathbb{\Phi}^{d-1}$ and $\Lambda_{\omega}$ with the standard $(d-1)$-dimensional Lebesgue measure. We set

$$
M(\omega, \xi)=\int_{-\infty}^{\infty}\langle A(t \omega+\xi), \omega\rangle \mathrm{d} t, \quad \omega \in \mathbb{S}^{d-1}, \xi \in \mathbb{R}^{d}
$$

Our main result is as follows.

Theorem 1.1. Let $V$, A satisfy (1.1). Then for any function $\varphi \in C(\mathbb{T})$ that vanishes in a neighborhood of the point 1 ,

$$
\lim _{k \rightarrow \infty} k^{-d+1} \operatorname{Tr} \varphi(S(k))=(2 \pi)^{-d+1} \int_{\Phi^{d}-1} \int_{\Lambda_{\omega}} \varphi\left(e^{i M(\omega, \xi)}\right) \mathrm{d} \xi \mathrm{d} \omega .
$$

Under the assumptions of Theorem 1.1, the operator $\varphi(S(k))$ has a finite rank, and so the trace in the left hand side exists; also, the integrand in the right hand side vanishes for all sufficiently large $|\xi|$, and so the integral is absolutely convergent.

The interesting feature of formula (1.4) is that its right hand side does not depend on the electric potential $V$; see the discussion at the end of Section 1.3.

\subsection{Discussion}

Weak convergence of measures. Theorem 1.1 can be rephrased in terms of weak convergence of the eigenvalue counting measures. Let us denote the eigenvalues of $S(k)$ by $e^{i \theta_{n}(k)}$, where $n \in \mathbb{N}$ and $\theta_{n}(k) \in[-\pi, \pi)$; as usual, the eigenvalues are counted with multiplicities taken into account. Let $\iota \subset \mathbb{T} \backslash\{1\}$ be any open arc separated away from 1; we set

$$
\begin{aligned}
& \mu_{k}(\iota)=\#\left\{n \in \mathbb{N}: e^{i \theta_{n}(k)} \in \iota\right\}, \quad k>0, \\
& \mu(\iota)=(2 \pi)^{-d+1} \int_{\Phi_{d}-1} \int_{\Pi_{\omega}(\iota)} \mathrm{d} \xi \mathrm{d} \omega, \quad \Pi_{\omega}(\iota)=\left\{\xi \in \Lambda_{\omega}: e^{i M(\omega, \xi)} \in \iota\right\},
\end{aligned}
$$


where \# represents the number of elements in a set. The measures $\mu_{k}, \mu$ can be alternatively defined by requiring that

$$
\begin{aligned}
\operatorname{Tr} \varphi(S(k)) & =\int_{\mathbb{T}} \varphi(z) \mathrm{d} \mu_{k}(z), \\
(2 \pi)^{-d+1} \int_{\Phi d-1} \int_{\Lambda_{\omega}} \varphi\left(e^{i M(\omega, \xi)}\right) \mathrm{d} \xi \mathrm{d} \omega & =\int_{\mathbb{T}} \varphi(z) \mathrm{d} \mu(z),
\end{aligned}
$$

for any continuous function $\varphi$ on $\mathbb{T}$ vanishing in a neighborhood of 1 . With this notation, Theorem 1.1 can be rephrased as the weak convergence

$$
k^{-d+1} \mu_{k} \longrightarrow \mu, \quad k \rightarrow \infty .
$$

The measure $\mu$ may be singular at 1 , i.e. $\mu(\mathbb{T} \backslash\{1\})$ may be infinite, but

$$
\int_{\mathbb{T}}|z-1|^{\ell} \mathrm{d} \mu(z)<+\infty, \quad \ell>(d-1) /(\rho-1) .
$$

Relation (1.9) follows from the elementary estimate on $M(\omega, \xi)$ which is a direct consequence of (1.1):

$$
|M(\omega, \xi)| \leq C(A)(1+|\xi|)^{1-\rho}, \quad \omega \in \mathbb{S}^{d-1}, \xi \in \Lambda_{\omega} .
$$

The case $\boldsymbol{A} \equiv \mathbf{0}$. In this case, the limiting measure $\mu$ vanishes, and in order to obtain non-trivial asymptotics of $\mu_{k}$, the problem requires an appropriate rescaling. By (1.2), the spectrum of $S(k)$ consists of eigenvalues which lie on an arc of length $O\left(k^{-1}\right)$ around 1. This suggests the following rescaled version of the problem: for an interval $\delta \subset \mathbb{R} \backslash\{0\}$ separated away from zero, set

$$
\tilde{\mu}_{k}(\delta)=\#\left\{n \in \mathbb{N}: k \theta_{n}(k) \in \delta\right\} .
$$

Then it turns out (see [6]) that

$$
k^{-d+1} \tilde{\mu}_{k} \longrightarrow \tilde{\mu} \quad \text { weakly as } k \rightarrow \infty,
$$

where the measure $\tilde{\mu}$ is defined as follows. Let

$$
X(\omega, \xi)=-\frac{1}{2} \int_{-\infty}^{\infty} V(t \omega+\xi) \mathrm{d} t, \quad \omega \in \mathbb{S}^{d-1}, \xi \in \Lambda_{\omega} ;
$$

then

$$
\tilde{\mu}(\delta)=(2 \pi)^{-d+1} \int_{\Phi^{d-1}} \int_{\widetilde{\Pi}_{\omega}(\iota)} \mathrm{d} \xi \mathrm{d} \omega, \quad \widetilde{\Pi}_{\omega}(\iota)=\left\{\xi \in \Lambda_{\omega}: X(\omega, \xi) \in \delta\right\},
$$

where $\delta \subset \mathbb{R} \backslash\{0\}$. 
Two and three dimensional cases. Let us discuss assumptions (1.1) in dimensions $d=2,3$. In dimension $d=3$, a magnetic vector-potential $A$ satisfying (1.1) can be constructed for any smooth magnetic field $B: \mathbb{R}^{3} \rightarrow \mathbb{R}^{3}(B=\operatorname{curl} A)$ such that $\operatorname{div} B=0$ and

$$
\left|\partial^{\alpha} B(x)\right| \leq C_{\alpha}\langle x\rangle^{-\rho-1-|\alpha|}, \quad \rho>1 .
$$

In dimension $d=2$, the magnetic field $B: \mathbb{R}^{2} \rightarrow \mathbb{R}$, in addition to (1.13), must satisfy the zero flux condition

$$
\Phi=\int_{\mathbb{R}^{2}} B(x) \mathrm{d} x=0, \quad B(x)=\frac{\partial A_{2}}{\partial x_{1}}-\frac{\partial A_{1}}{\partial x_{2}} .
$$

See e.g. [20], Section 5.1, for the details of this construction both for $d=2$ and $d=3$.

The zero flux condition is unavoidable in the following sense. Let $B: \mathbb{R}^{2} \rightarrow \mathbb{R}$ be such that it satisfies the estimates (1.13) but the flux $\Phi \neq 0$. Then any magnetic vectorpotential $A(x)$ for this field will necessarily fail to be short-range (i.e. (1.1) fails) but one can construct $A(x)$ (the "transversal gauge") with the behavior $|A(x)| \sim|x|^{-1}$ as $|x| \rightarrow \infty$. In this case the scattering theory for $H_{0}, H$ can still be constructed, but the difference $S(k)-I$ will not be compact, see [20] for a detailed discussion and a description of the essential spectrum of $S(k)$. A particularly well known example of this is the Aharonov-Bohm effect [2]. Thus, in this case the measure $\mu_{k}$ cannot even be defined and the question of the spectral asymptotics of the scattering matrix cannot be approached in the same way as in Theorem 1.1.

Gauge invariance. The scattering matrix is gauge invariant in the class of shortrange magnetic vector-potentials. More precisely, if

$$
\widetilde{A}(x)=A(x)+\nabla \varphi(x), \quad \text { where } \varphi(x) \longrightarrow 0 \text { as }|x| \rightarrow \infty,
$$

then the scattering matrix $\widetilde{S}(k)$ associated with the pair $\widetilde{H}=(i \nabla+\widetilde{A})^{2}+V$, $H_{0}=-\Delta$, coincides with $S(k)$. See [13] and [20] for further details and for an interesting discussion of examples when $S(k)$ is not gauge invariant (when $\varphi(x)$ is homogeneous in $|x|$ of order zero). An inspection shows that $M(\omega, \xi)$ is gauge invariant under the gauge transformations of the class (1.15).

Related work. Although the study of various aspects of asymptotic distribution of eigenvalues of differential operators has become a well-developed industry, very little attention in mathematical literature has been devoted to analogous questions for the scattering matrix. There has been, of course, much work on the "average" characteristics of the scattering matrix $S(k)$ : on the scattering phase $\arg \operatorname{det} S(k)$ and on the total scattering cross section; but the study of the distribution of individual eigenvalues of $S(k)$ has been less popular. We are only aware of mathematical works [11], [4], [5], [22], and [7] on this subject. In [11], the asymptotic behavior 
of the phases $\theta_{n}(k)$ was determined for a fixed $k$ and $n \rightarrow \infty$ for potentials $V$ with compact support and $A \equiv 0$. In [4], the same problem was considered in the case of potentials $V$ with a power asymptotics at infinity. In [5], this question was studied in the presence of a periodic background potential. In [22], the spacing between the phases $\theta_{n}(k)$ was studied in a rather special model of scattering on manifolds.

In [7], a semiclassical asymptotics of phase shifts is considered for the Schrödinger operator with a spherical symmetric potential and equidistribution of these phase shifts is proven.

In the physics community, the question of asymptotic distribution of eigenvalues of the scattering matrix has been addressed; see e.g. the works by U. Smilansky and his collaborators [9] on the eigenvalue statistics of $S(k)$ for obstacle scattering.

The limiting measure $\mu$ arises via integration over straight lines, i.e. over the trajectories of the free dynamics, see (1.3). Similar asymptotic formulas are known in other problems, involving the discrete spectrum of differential and pseudodifferential operators; see [18], [16], [17], and [12].

1.3. Key steps of the proof of Theorem 1.1. Our proof is heavily based on the results of [19]. In [19], D. Yafaev suggested a high energy asymptotic expansion for the integral kernel of the scattering matrix $S(k)$. This expansion is constructed via approximate scattering solutions to the stationary Schrödinger equation

$$
H u=k^{2} u \text {. }
$$

We recall this construction in Section 2. Using Yafaev's expansion, it is easy to represent the scattering matrix $S(k)$ (modulo a negligible error) as a semiclassical pseudodifferential operator ( $\Psi D O)$ on the sphere with the semiclassical parameter $h=k^{-1}$ and the principal symbol $e^{i M(\omega, \xi)}$. This representation is already present, in a somewhat different form, in [19]; we re-derive it in Section 2 in the form convenient for our purposes (see Lemma 2.5). After this, using the standard pseudodifferential techniques, we prove (see Lemma 3.1) the asymptotic formula

$$
\begin{aligned}
& \lim _{k \rightarrow \infty} k^{-d+1} \operatorname{Tr}(S(k)-I)^{\ell_{1}}\left(S(k)^{*}-I\right)^{\ell_{2}} \\
& \quad=(2 \pi)^{-d+1} \int_{\Phi d-1} \int_{\Lambda_{\omega}}\left(e^{i M(\omega, \xi)}-I\right)^{\ell_{1}}\left(e^{-i M(\omega, \xi)}-I\right)^{\ell_{2}} \mathrm{~d} \xi \mathrm{d} \omega
\end{aligned}
$$

for any integers $\ell_{1} \geq 0, \ell_{2} \geq 0$ such that the sum $\ell_{1}+\ell_{2}$ is sufficiently large. From here it is easy to derive the main result by an application of the Weierstrass approximation theorem; this is done in Section 3.

The difference between the case $A \equiv 0$ and the general case can be understood as follows. As mentioned above, the scattering matrix can be approximated by a $\Psi \mathrm{DO}$ on the sphere with a symbol which depends on $k$. The leading term of the asymptotic expansion of this symbol in powers of $k^{-1}$ is $e^{i M(\omega, \xi)}$ (if $A \not \equiv 0$ ); this term involves only the magnetic vector potential $A$. The electric potential turns up only in the next 
term of the expansion, which (if $A \equiv 0$ ) has the form $i k^{-1} X(\omega, \xi)$, see (1.12). Of course, this is related to the fact that the magnetic potential is a coefficient of the differential operator of the order 1 , whereas the electric potential is a perturbation of the order 0 .

\section{The scattering matrix as a $\Psi D O$ on the sphere.}

2.1. The scattering matrix. Let us briefly recall the definition of the scattering matrix; for the details, we refer to any textbook on scattering theory, e.g. [21]. It is one of the fundamental facts of scattering theory that under the conditions (1.1), the wave operators

$$
W_{ \pm}=\underset{t \rightarrow \pm \infty}{s-\lim _{t \rightarrow \infty}} e^{i t H} e^{-i t H_{0}}
$$

exist and are complete. The scattering operator $\mathbf{S}=W_{+}^{*} W_{-}$is unitary in $L^{2}\left(\mathbb{R}^{d}\right)$ and commutes with $H_{0}$. Let $F: L^{2}\left(\mathbb{R}^{d}\right) \rightarrow L^{2}\left((0, \infty) ; L^{2}\left(\mathbb{\$}^{d-1}\right)\right)$ be the unitary operator

$$
(F u)(k, \omega)=\frac{1}{\sqrt{2}} k^{(d-2) / 2} \hat{u}(k \omega), \quad k>0, \omega \in \mathbb{S}^{d-1},
$$

where $\hat{u}$ is the usual (unitary) Fourier transform of $u$. The operator $F$ diagonalises $H_{0}$, i.e.

$$
\left(F H_{0} u\right)(k, \omega)=k^{2}(F u)(k, \omega), \quad u \in C_{0}^{\infty}\left(\mathbb{R}^{d}\right) .
$$

The commutation relation $\mathbf{S} H_{0}=H_{0} \mathbf{S}$ implies that $F$ also diagonalises $\mathbf{S}$, i.e.

$$
(F \mathbf{S} u)(k, \cdot)=S(k)(F u)(k, \cdot),
$$

where $S(k): L^{2}\left(\Phi^{d-1}\right) \rightarrow L^{2}\left(\Phi^{d-1}\right)$ is the unitary operator known as the (on-shell) scattering matrix.

2.2. Pseudodifferential operators on the sphere. For every $\omega \in \mathbb{S}^{d-1}$, we identify the cotangent space $T_{\omega}^{*} \mathbb{\Phi}^{d-1}$ with the plane $\Lambda_{\omega}=\left\{x \in \mathbb{R}^{d}:\langle x, \omega\rangle=0\right\}$ in a standard way. For a symbol $\sigma \in C_{0}^{\infty}\left(T^{*} \mathbb{\$}^{d-1}\right)$ and a semiclassical parameter $h \in$ $(0,1)$, the semiclassical $\Psi \mathrm{DOO} \mathrm{p}_{h}[\sigma]$ in $L^{2}\left(\$^{d-1}\right)$ is defined via its integral kernel

$$
\mathrm{Op}_{h}[\sigma]\left(\omega, \omega^{\prime}\right)=(2 \pi h)^{-d+1} \int_{\Lambda_{\omega}} e^{-i\left\langle\omega-\omega^{\prime}, \xi\right\rangle / h} \sigma(\omega, \xi) \mathrm{d} \xi,
$$

where $\omega, \omega^{\prime} \in \mathbb{S}^{d-1}$. This definition can be extended in a standard way to symbols $\sigma$ satisfying

$$
\left|\partial_{\xi}^{\alpha} \partial_{\omega}^{\beta} \sigma(\omega, \xi)\right| \leq C_{\alpha \beta}\langle\xi\rangle^{-m-|\alpha|}, \quad \omega \in \mathbb{S}^{d-1}, \xi \in \Lambda_{\omega},
$$


for some $m \in \mathbb{R}$ and all multi-indices $\alpha, \beta$. We will only be interested in the case $m>0$; then by the Calderon-Villancourt theorem (see e.g. [15]) combined with a scaling argument, $\mathrm{Op}_{h}[\sigma]$ is bounded and

$$
\sup _{0<h<1}\left\|\mathrm{Op}_{h}[\sigma]\right\| \leq C(\sigma) .
$$

Next, if $\sigma$ satisfies (2.2) with $m>d-1$, then (see e.g. [14] and [8]) $\mathrm{Op}_{h}[\sigma]$ is trace class and its trace can be computed by integrating the kernel (2.1) over the diagonal:

$$
\operatorname{Tr}\left(\mathrm{Op}_{h}[\sigma]\right)=(2 \pi h)^{-d+1} \int_{\Phi^{d-1}} \int_{\Lambda_{\omega}} \sigma(\omega, \xi) \mathrm{d} \xi \mathrm{d} \omega .
$$

We will also be interested in symbols that depend on $h$. For $m>0$, let $\oint^{m}$ be the class of $C^{\infty}$-smooth symbols $\sigma=\sigma(\omega, \xi, h), h \in(0,1)$, satisfying the estimate (2.2) uniformly in $h \in(0,1)$ for all multi-indices $\alpha, \beta$. We will need a standard statement about the leading term spectral asymptotics of a semiclassical UDO:

Proposition 2.1. Let $m>0$ and let $\sigma \in \S^{m}$ be a symbol that admits the representation

$$
\sigma=\sigma_{0}+h \sigma_{1}
$$

with $\sigma_{0}, \sigma_{1} \in \mathcal{S}^{m}$ and $\sigma_{0}$ independent of $h$. Then for any non-negative integers $\ell_{1}$, $\ell_{2}$ such that $\ell_{1}+\ell_{2}>\frac{d-1}{m}$, the operator $\left(\mathrm{Op}_{h}[\sigma]\right)^{\ell_{1}}\left(\left(\mathrm{Op}_{h}[\sigma]\right)^{*}\right)^{\ell_{2}}$ belongs to the trace class and

$$
\begin{gathered}
\lim _{h \rightarrow+0}(2 \pi h)^{d-1} \operatorname{Tr}\left(\left(\mathrm{Op}_{h}[\sigma]\right)^{\ell_{1}}\left(\left(\mathrm{Op}_{h}[\sigma]\right)^{*}\right)^{\ell_{2}}\right) \\
\quad=\int_{\Phi d-1} \int_{\Lambda_{\omega}} \sigma_{0}(\omega, \xi)^{\ell_{1}} \overline{\sigma_{0}(\omega, \xi)^{\ell_{2}}} \mathrm{~d} \xi \mathrm{d} \omega .
\end{gathered}
$$

Sketch of proof. First suppose that $m>d-1$ and $\ell_{1}=1, \ell_{2}=0$. Then $\mathrm{Op}_{h}[\sigma]$ is trace class and the asymptotics (2.6) follow by substituting the representation (2.5) into (2.4). In the general case, using local coordinates on the sphere, iterating a standard composition formula for $\Psi \mathrm{DO}$ in $L^{2}\left(\mathbb{R}^{d-1}\right)$, and using the formula for the symbol of the adjoint operator, we obtain that for any $N>0$,

$$
\left(\mathrm{Op}_{h}[\sigma]\right)^{\ell_{1}}\left(\left(\mathrm{Op}_{h}[\sigma]\right)^{*}\right)^{\ell_{2}}=\mathrm{Op}_{h}[\varkappa]+R_{N}(h),
$$

where

(i) the symbol $\varkappa \in \mathcal{S}^{m \ell_{1}+m \ell_{2}}$ can be represented as

$$
\varkappa=\varkappa_{0}+h \varkappa_{1}^{(N)}
$$

with $\varkappa_{0}, \varkappa_{1}^{(N)} \in \mathcal{S}^{m \ell_{1}+m \ell_{2}}, \varkappa_{0}$ is independent of $h$ and

$$
\varkappa_{0}(\omega, \xi)=\sigma_{0}(\omega, \xi)^{\ell_{1}} \overline{\sigma_{0}(\omega, \xi)^{\ell_{2}}} ;
$$


(ii) $R_{N}(h)$ is an operator with the integral kernel in $C^{N}\left(\mathbb{S}^{d-1} \times \mathbb{S}^{d-1}\right)$ and the $C^{N}$-norm of $R_{N}(h)$ is $O\left(h^{N}\right)$ as $h \rightarrow 0$.

This reduces the problem to the case $\ell_{1}=1, \ell_{2}=0$.

Remark. In the situation we are interested in, the representation (2.5) arises as a corollary of the asymptotic expansion

$$
\sigma \sim \sum_{j=0}^{\infty} h^{j} \sigma_{j}, \quad \sigma_{j} \in S^{m}
$$

but we are only interested in the first term of this expansion.

In our construction, the $\Psi$ DO will be defined in terms of their amplitudes rather than their symbols. Thus, we need a statement which is standard in the UDO theory (see e.g. [14]).

Proposition 2.2. Let $m>0$, and let $b=b\left(\omega, \omega^{\prime}, \xi, h\right)$ be a smooth function of the variables $(\omega, \xi) \in T^{*} \mathbb{S}^{d-1}, \omega^{\prime} \in \mathbb{S}^{d-1}$ and $h \in(0,1)$. Assume that $b$ satisfies the estimates

$$
\left|\partial_{\xi}^{\alpha} \partial_{\omega}^{\beta} \partial_{\omega^{\prime}}^{\gamma} b\left(\omega, \omega^{\prime}, \xi, h\right)\right| \leq C_{\alpha \beta \gamma}\langle\xi\rangle^{-m-|\alpha|}
$$

for all multi-indices $\alpha, \beta, \gamma$ uniformly in $h \in(0,1)$. Then for any $N>0$, the operator with the integral kernel

$$
(2 \pi h)^{-d+1} \int_{\Lambda_{\omega}} e^{-i\left\langle\omega-\omega^{\prime}, \xi\right\rangle / h} b\left(\omega, \omega^{\prime}, \xi, h\right) \mathrm{d} \xi
$$

can be represented as $\mathrm{Op}_{h}[\sigma]+R_{N}(h)$, where the following conditions are met:

(i) the symbol $\sigma$ can be written as $\sigma=\sigma_{0}+h \sigma_{1}$ with $\sigma_{0}, \sigma_{1} \in \mathcal{S}^{m}$ and

$$
\sigma_{0}(\omega, \xi, h)=b(\omega, \omega, \xi, h) \text {. }
$$

(ii) the operator $R_{N}(h)$ has the integral kernel in $C^{N}\left(\mathbb{S}^{d-1} \times \mathbb{S}^{d-1}\right)$ with $C^{N_{-n o r m}}$ satisfying $O\left(h^{N}\right)$ as $h \rightarrow 0$.

2.3. Approximate solutions to the Schrödinger equation. Here we recall the construction of approximate solutions to the Schrödinger equation $H u=k^{2} u$ from [19]. The solutions $u$ are sought as functions

$$
u=u(x, p), \quad x \in \mathbb{R}^{d}, p \in \mathbb{R}^{d},|p|=k .
$$

We denote $\hat{p}=p|p|^{-1} \in \mathbb{S}^{d-1}$. Substituting the ansatz

$$
u(x, p)=e^{i \Theta(x, p)} v(x, p)
$$


into the Schrödinger equation $H u=k^{2} u$, by a standard procedure (see e.g. [10], Section 7) we obtain the eikonal equation

$$
|\nabla \Theta(x)-A(x)|^{2}+V(x)=k^{2}
$$

for the phase function $\Theta$ and the transport equation

$$
-2 i\langle\nabla \Theta, \nabla v\rangle+2 i\langle A, \nabla v\rangle-\Delta v+(-i \Delta \Theta+i \operatorname{div} A) v=0
$$

for the amplitude function $v$. The approximate solution to the eikonal equation is constructed as

$$
\begin{aligned}
& \Theta_{ \pm}(x, p)=\langle x, p\rangle+\varphi_{ \pm}(x, \hat{p}), \\
& \varphi_{ \pm}(x, \hat{p})=\mp \int_{0}^{\infty}\langle A(x \pm t \hat{p}), \hat{p}\rangle \mathrm{d} t .
\end{aligned}
$$

Next, for a given $N \in \mathbb{N}$, the approximate solution $v_{ \pm}^{(N)}$ to the transport equation is constructed as

$$
v_{ \pm}^{(N)}(x, p)=\sum_{n=0}^{N}(2 i k)^{-n} v_{n}^{( \pm)}(x, \hat{p}),
$$

where $v_{0}^{( \pm)}(x, \hat{p}) \equiv 1$ and the coefficients $v_{n}^{( \pm)}$are determined via an explicit iterative procedure:

$$
v_{n+1}^{( \pm)}(x, \hat{p})=\mp \int_{0}^{\infty} f_{n}^{( \pm)}(x \pm t \hat{p}, \hat{p}) \mathrm{d} t,
$$

where

$f_{n}^{( \pm)}=2 i\left\langle A-\nabla \varphi_{ \pm}, \nabla v_{n}^{( \pm)}\right\rangle-\Delta v_{n}^{( \pm)}+\left(\left|\nabla \varphi_{ \pm}\right|^{2}-2\left\langle A, \nabla \varphi_{ \pm}\right\rangle+V_{1}-i \Delta \varphi_{ \pm}\right) v_{n}^{( \pm)}$,

and

$$
V_{1}=V+|A|^{2}+i \operatorname{div} A .
$$

The functions $\varphi_{ \pm}$and $v_{ \pm}^{(N)}$ solve the eikonal and transport equations up to error terms that can be explicitly controlled; we do not need the precise statement here, see [19] for the details. Here we need $\varphi_{ \pm}$and $v_{ \pm}^{(N)}$ simply as "building blocks" for the approximation to the scattering amplitude, which is given in the next subsection.

When considering the functions $\varphi_{ \pm}$and $v_{n}^{( \pm)}$, we will always exclude a conical neighborhood of the direction $\hat{x}=-\hat{p}$ (for the sign "+") or $\hat{x}=\hat{p}$ (for the sign "-"). Outside these neighborhoods, the functions $\varphi_{ \pm}$and $v_{n}^{( \pm)}, n \geq 1$, decay at infinity in the $x$-variable. More precisely, the following statement is proven in [19].

Proposition 2.3. [19] Let assumption (1.1) hold and let $x \in \mathbb{R}^{d}, \omega \in \mathbb{S}^{d-1}$ be such that $\pm\langle\hat{x}, \omega\rangle \geq-1+\varepsilon$ for some $\varepsilon>0$. Then the functions $\varphi_{ \pm}$and $v_{n}^{( \pm)}, n \geq 1$, 
satisfy the estimates

$$
\begin{aligned}
\left|\partial_{x}^{\alpha} \partial_{\omega}^{\beta} \varphi_{ \pm}(x, \omega)\right| & \leq C_{\alpha \beta}\langle x\rangle^{1-\rho-|\alpha|}, \\
\left|\partial_{x}^{\alpha} \partial_{\omega}^{\beta} v_{n}^{( \pm)}(x, \omega)\right| & \leq C_{\alpha \beta}\langle x\rangle^{-n-|\alpha|},
\end{aligned}
$$

for all multi-indices $\alpha, \beta$.

We will write

$$
u_{ \pm}^{(N)}(x, p)=u_{ \pm}(x, p)=e^{i \Theta_{ \pm}(x, p)} v_{ \pm}^{(N)}(x, p) .
$$

2.4. Approximation for the scattering amplitude. Here we recall the approximation to the scattering amplitude obtained in [19]. It is known (see e.g. [1]) that off the diagonal $\omega=\omega^{\prime}$, the integral kernel $s\left(\omega, \omega^{\prime} ; k\right)$ of the scattering matrix $S(k)$ is a $C^{\infty}$-smooth function of $\omega, \omega^{\prime} \in \mathbb{S}^{d-1}$ and it tends to zero faster than any power of $k^{-1}$ as $k \rightarrow \infty$. Thus, it suffices to describe the structure of $s\left(\omega, \omega^{\prime} ; k\right)$ in a neighborhood of the diagonal $\omega=\omega^{\prime}$. Fix some $\delta \in(0,1)$; for an arbitrary point $\omega_{0} \in \mathbb{S}^{d-1}$, let $\Omega\left(\omega_{0}\right) \subset \mathbb{S}^{d-1}$ be the conical neighborhood of $\omega_{0}$ given by

$$
\Omega\left(\omega_{0}\right)=\left\{\omega \in \mathbb{S}^{d-1}:\left\langle\omega, \omega_{0}\right\rangle>\delta\right\} .
$$

Let $u_{ \pm}$be as in (2.14). We set

$$
\partial_{\omega_{0}} u=\left\langle\nabla u, \omega_{0}\right\rangle
$$

where the gradient of $u=u(x, p)$ is taken in the $x$ variable. For $\omega, \omega^{\prime} \in \Omega\left(\omega_{0}\right)$, define

$$
\begin{aligned}
s_{0}^{(N)}\left(\omega, \omega^{\prime} ; k\right)=- & i \pi k^{d-2}(2 \pi)^{-d} \\
\times & \left(\int_{\Lambda_{\omega_{0}}} \overline{\left[u_{+}^{(N)}(x, k \omega)\right.}\left(\partial_{\omega_{0}} u_{-}^{(N)}\right)\left(x, k \omega^{\prime}\right)\right. \\
& \left.-2 i \int_{\Lambda_{\omega_{0}}}\left\langle A(x), \omega_{0}\right\rangle \overline{u_{+}^{(N)}(x, k \omega)} u_{-}^{(N)}\left(x, k \omega^{\prime}\right) \mathrm{d} x\right) .
\end{aligned}
$$

The integrals in (2.16) do not converge absolutely and should be understood as oscillatory integrals. In other words, (2.16) should be understood as a distribution on $\Omega\left(\omega_{0}\right) \times \Omega\left(\omega_{0}\right)$.

Proposition 2.4. [19] For any $q \in \mathbb{N}$ there exists $N=N(q) \in \mathbb{N}$ such that for any $\omega_{0} \in \mathbb{S}^{d-1}$, the kernel

$$
\tilde{s}^{(N)}\left(\omega, \omega^{\prime} ; k\right)=s\left(\omega, \omega^{\prime} ; k\right)-s_{0}^{(N)}\left(\omega, \omega^{\prime} ; k\right)
$$

belongs to the class $C^{q}\left(\Omega\left(\omega_{0}\right) \times \Omega\left(\omega_{0}\right)\right)$, and its $C^{q}$-norm is $O\left(k^{-q}\right)$ as $k \rightarrow \infty$. 
2.5. The scattering matrix as a $\Psi \mathrm{DO}$ on the sphere. Below we represent the scattering matrix $S(k)$ as a semiclassical $\Psi D O$ on the sphere. The semiclassical parameter is $h=k^{-1}$. The idea to represent the scattering matrix as a UDO on the sphere goes back to [4]. The statements almost identical to Lemma 2.5 can be found in [19], Propositions 6.1 and 6.4, and [21], Section 8.4, but for technical reasons these statements are not sufficient for our purposes. A related work (but written from a very different viewpoint) is [3], where the scattering matrix is represented as a Fourier integral operator corresponding to the classical scattering relation.

Lemma 2.5. Let assumptions (1.1) hold, and let $m=\min \{1, \rho-1\}$. For any $q \in \mathbb{N}$, the scattering matrix can be written as

$$
S(k)=I+\mathrm{Op}_{k^{-1}}[\sigma]+R_{q}(k),
$$

where

(i) the symbol $\sigma$ can be represented as

$$
\sigma=\sigma_{0}+k^{-1} \sigma_{1}
$$

with $\sigma_{0}, \sigma_{1} \in \mathcal{S}^{m}$ and

$$
\sigma_{0}(\omega, \xi)=\exp (i M(\omega, \xi))-1 ;
$$

(ii) the operator $R_{q}(k)$ has an integral kernel in the class $C^{q}\left(\$^{d-1} \times \$^{d-1}\right)$ and its $C^{q}$-norm is $O\left(k^{-q}\right)$ as $k \rightarrow \infty$.

Proof. 1) Let $\psi_{1}, \psi_{2} \in C^{\infty}\left(\mathbb{S}^{d-1}\right)$ be functions with disjoint supports. Then $\psi_{1} S(k) \psi_{2}$ has a $C^{\infty}$-smooth integral kernel which decays faster than any power of $k^{-1}$ as $k \rightarrow \infty$, see e.g. [1]. The same comment applies to $\psi_{1} \mathrm{Op}_{k^{-1}}[a] \psi_{2}$ with $a \in \varsigma^{m}$. This shows that using a sufficiently fine partition of unity on the sphere, one easily reduces the problem to approximating the integral kernel of $S(k)$ locally in any conical neighborhood $\Omega\left(\omega_{0}\right)$, see (2.15). Thus, we can use Proposition 2.4.

2) Let us rearrange the integrand in (2.16). Denote

$$
\begin{aligned}
& w_{ \pm}(x, p)=e^{i \varphi_{ \pm}(x, \hat{p})} v_{ \pm}^{(N)}(x, p), \\
& \tilde{w}_{ \pm}(x, p)=k e^{i \varphi_{ \pm}(x, \hat{p})}\left(v_{ \pm}^{(N)}(x, p)-1\right),
\end{aligned}
$$

so that from (2.14),

$$
\begin{aligned}
u_{ \pm}(x, k \omega) & =e^{i k\langle x, \omega\rangle} w_{ \pm}(x, k \omega) \\
= & e^{i k\langle x, \omega\rangle}\left(e^{i \varphi_{ \pm}(x, \omega)}+k^{-1} \tilde{w}_{ \pm}(x, k \omega)\right), \\
\left(\partial_{\omega_{0}} u_{ \pm}\right)(x, k \omega)= & e^{i k\langle x, \omega\rangle}\left[i k\left\langle\omega_{0}, \omega\right\rangle e^{i \varphi_{ \pm}}(x, \omega)\right. \\
& \left.+i\left\langle\omega_{0}, \omega\right\rangle \tilde{w}_{ \pm}(x, k \omega)+\left(\partial_{\omega_{0}} w_{ \pm}\right)(x, k \omega)\right] .
\end{aligned}
$$


Now some elementary algebra shows that formula (2.16) can be rewritten as

$$
s_{0}^{(N)}\left(\omega, \omega^{\prime} ; k\right)=\left(\frac{k}{2 \pi}\right)^{d-1} \int_{\Lambda_{\omega_{0}}} e^{-i k\left\langle\omega-\omega^{\prime}, x\right\rangle} a\left(\omega, \omega^{\prime}, x\right) \mathrm{d} x,
$$

where

$$
a\left(\omega, \omega^{\prime}, x\right)=\frac{1}{2}\left\langle\omega_{0}, \omega+\omega^{\prime}\right\rangle \exp \left(i \varphi_{-}\left(x, \omega^{\prime}\right)-i \varphi_{+}(x, \omega)\right)+k^{-1} a_{1}\left(\omega, \omega^{\prime}, x\right),
$$

and

$$
\begin{aligned}
2 a_{1}\left(\omega, \omega^{\prime}, x\right)=\langle & \left., \omega_{0}\right\rangle\left(e^{-i \varphi_{+}(x, \omega)} \tilde{w}_{-}\left(x, k \omega^{\prime}\right)+\overline{\tilde{w}_{+}(x, k \omega)} w_{-}\left(x, k \omega^{\prime}\right)\right) \\
& +\left\langle\omega^{\prime}, \omega_{0}\right\rangle\left(e^{i \varphi-\left(x, \omega^{\prime}\right)} \overline{\tilde{w}_{+}(x, k \omega)}+\overline{w_{+}(x, k \omega)} \tilde{w}_{-}\left(x, k \omega^{\prime}\right)\right) \\
& +i \overline{\left(\partial_{\omega_{0}} w_{+}\right)(x, k \omega)} w_{-}\left(x, k \omega^{\prime}\right)-i \overline{w_{+}(x, k \omega)}\left(\partial_{\omega_{0}} w_{-}\right)\left(x, k \omega^{\prime}\right) \\
& -2\left\langle A(x), \omega_{0}\right\rangle \overline{w_{+}(x, k \omega)} w_{-}\left(x, k \omega^{\prime}\right) .
\end{aligned}
$$

Note that the choice $a\left(\omega, \omega^{\prime}, x\right)=\frac{1}{2}\left\langle\omega+\omega^{\prime}, \omega_{0}\right\rangle$ in (2.22) yields a $\delta$-function on the sphere. Thus, we can write

$$
\begin{aligned}
& s_{0}^{(N)}\left(\omega, \omega^{\prime} ; k\right)-\delta\left(\omega-\omega^{\prime}\right) \\
& \quad=\left(\frac{k}{2 \pi}\right)^{d-1} \int_{\Lambda_{\omega_{0}}} e^{-i k\left\langle\omega-\omega^{\prime}, x\right\rangle}\left(a_{0}+k^{-1} a_{1}\right)\left(\omega, \omega^{\prime}, x\right) \mathrm{d} x,
\end{aligned}
$$

where

$$
a_{0}\left(\omega, \omega^{\prime}, x\right)=\frac{1}{2}\left\langle\omega_{0}, \omega+\omega^{\prime}\right\rangle\left(\exp \left(i \varphi_{-}\left(x, \omega^{\prime}\right)-i \varphi_{+}(x, \omega)\right)-1\right) .
$$

3) Let us change variables in the integral (2.25). Instead of integrating over $x \in \Lambda_{\omega_{0}}$, we shall integrate over $\xi \in \Lambda_{\omega}$, where

$$
x=\xi-\frac{\left\langle\xi, \omega_{0}\right\rangle}{\left\langle\omega+\omega^{\prime}, \omega_{0}\right\rangle}\left(\omega+\omega^{\prime}\right)
$$

Recall that $\omega, \omega^{\prime} \in \Omega\left(\omega_{0}\right)$, and so the denominator in (2.27) does not vanish. An inspection shows that

$$
\left\langle x, \omega-\omega^{\prime}\right\rangle=\left\langle\xi, \omega-\omega^{\prime}\right\rangle .
$$

Thus, we obtain

$$
\begin{aligned}
& s_{0}^{(N)}\left(\omega, \omega^{\prime} ; k\right)-\delta\left(\omega-\omega^{\prime}\right) \\
& \quad=\left(\frac{k}{2 \pi}\right)^{d-1} \int_{\Lambda_{\omega}} e^{-i k\left\langle\omega-\omega^{\prime}, \xi\right\rangle} b\left(\omega, \omega^{\prime}, \xi\right) \mathrm{d} \xi,
\end{aligned}
$$


where $b=b_{0}+k^{-1} b_{1}$ with

$$
b_{j}\left(\omega, \omega^{\prime}, \xi\right)=J\left(\omega, \omega^{\prime}\right) a_{j}\left(\omega, \omega^{\prime}, x(\xi)\right)
$$

and $J\left(\omega, \omega^{\prime}\right)$ denotes the Jacobian of the linear map (2.27) considered as a map from $\Lambda_{\omega}$ to $\Lambda_{\omega_{0}}$. It is easy to see that $J\left(\omega, \omega^{\prime}\right)$ is a smooth function of $\omega, \omega^{\prime} \in \Omega\left(\omega_{0}\right)$ and

$$
J(\omega, \omega)=\left\langle\omega, \omega_{0}\right\rangle^{-1} .
$$

4) The right hand side of (2.28) is a semiclassical $\Psi D O$ with the amplitude $b$ and a semiclassical parameter $h=k^{-1}$, see (2.8). In order to complete the proof, by Proposition 2.2 if suffices to check the estimates

$$
\left|\partial_{x}^{\alpha} \partial_{\omega}^{\beta} \partial_{\omega^{\prime}}^{\gamma} b_{j}\left(\omega, \omega^{\prime}, \xi\right)\right| \leq C_{\alpha \beta \gamma}\langle\xi\rangle^{-m-|\alpha|},
$$

for $j=0,1$ and all multi-indices $\alpha, \beta, \gamma$ uniformly over $k \geq 1$, and to check the identity

$$
b_{0}(\omega, \omega, \xi)=\exp (i M(\omega, \xi))-1 .
$$

Let us first check (2.32). Recalling the definition (1.3) of $M$ and the definition (2.10) of $\varphi_{ \pm}$, we get

$$
M(\omega, \xi)=\varphi_{-}(\xi, \omega)-\varphi_{+}(\xi, \omega) .
$$

From this and (2.26), (2.29) and (2.30), we obtain

$$
b_{0}(\omega, \omega, \xi)=J(\omega, \omega) a_{0}(\omega, \omega, x(\xi))=\exp (i M(\omega, x(\xi)))-1,
$$

where $x(\xi)$ is the linear map (2.27). Next, by the definition of the map $x(\xi)$, for $\omega=\omega^{\prime}$ it takes the form $x(\xi)=\xi+c \omega$, and by the definition of the function $M$ we have

$$
M(\omega, \xi+c \omega)=M(\omega, \xi) .
$$

Thus, we obtain (2.32).

5) It remains to check that the estimates (2.31) are satisfied. This is essentially a consequence of Proposition 2.3; let us check this. Recalling that $m=\min \{1, \rho-1\}$ and using the estimates (2.12) and (2.13), we obtain

$$
\begin{aligned}
\left|\partial_{x}^{\alpha} \partial_{\omega}^{\beta} \varphi_{ \pm}(x, \omega)\right| & \leq C_{\alpha \beta}\langle x\rangle^{-m-|\alpha|}, \\
\left|\partial_{x}^{\alpha} \partial_{\omega}^{\beta} \tilde{w}_{ \pm}(x, k \omega)\right| & \leq C_{\alpha \beta}\langle x\rangle^{-m-|\alpha|}, \\
\left|\partial_{x}^{\alpha} \partial_{\omega}^{\beta}\left(\partial_{\omega_{0}} w_{ \pm}\right)(x, k \omega)\right| & \leq C_{\alpha \beta}\langle x\rangle^{-m-|\alpha|}, \\
\left|\partial_{x}^{\alpha} \partial_{\omega}^{\beta} w_{ \pm}(x, k \omega)\right| & \leq C_{\alpha \beta}\langle x\rangle^{-|\alpha|},
\end{aligned}
$$

where all the estimates are uniform in $k \geq 1$. It follows that $a_{0}$ and $a_{1}$, defined by (2.26) and (2.24), respectively, satisfy

$$
\left|\partial_{x}^{\alpha} \partial_{\omega}^{\beta} \partial_{\omega^{\prime}}^{\gamma} a_{j}\left(\omega, \omega^{\prime}, x\right)\right| \leq C_{\alpha \beta}\langle x\rangle^{-m-|\alpha|}
$$

uniformly in $k \geq 1$. Now from (2.27), (2.29), and (2.33) by an elementary calculation we obtain (2.31). 


\section{Proof of Theorem 1.1}

\subsection{The case of a monomial $\varphi$}

Lemma 3.1. Assume the hypothesis of Theorem 1.1. Then for any integers $\ell_{1} \geq 0$, $\ell_{2} \geq 0$ such that $\ell_{1}+\ell_{2}>(d-1) / m, m=\min \{1, \rho-1\}$, asymptotic formula $(1.16)$ holds true.

Proof. By Lemma 2.5, we have

$$
(S(k)-I)^{\ell_{1}}\left(S(k)^{*}-I\right)^{\ell_{2}}=\left(\mathrm{Op}_{k^{-1}}[\sigma]+R_{q}(k)\right)^{\ell_{1}}\left(\left(\mathrm{Op}_{k^{-1}}[\sigma]\right)^{*}+R_{q}(k)^{*}\right)^{\ell_{2}},
$$

where $\sigma, R_{q}(k)$ are as described in Lemma 2.5. Expanding the brackets in (3.1), we obtain

$$
(S(k)-I)^{\ell_{1}}\left(S(k)^{*}-I\right)^{\ell_{2}}=\left(\mathrm{Op}_{k^{-1}}[\sigma]\right)^{\ell_{1}}\left(\left(\mathrm{Op}_{k^{-1}}[\sigma]\right)^{*}\right)^{\ell_{2}}+Q_{q}(k),
$$

where $Q_{q}(k)$ is the sum of the products of operators, to be estimated below. For the first term in the right hand side in (3.2), by Proposition 2.1, we have

$$
\begin{aligned}
\lim _{k \rightarrow \infty} & \left(\frac{k}{2 \pi}\right)^{-d+1} \operatorname{Tr}\left(\left(\mathrm{Op}_{k^{-1}}[\sigma]\right)^{\ell_{1}}\left(\left(\mathrm{Op}_{k^{-1}}[\sigma]\right)^{*}\right)^{\ell_{2}}\right) \\
= & \int_{\Phi^{d}-1} \int_{\Lambda_{\omega}}\left(e^{i M(\omega, \xi)}-1\right)^{\ell_{1}}\left(e^{-i M(\omega, \xi)}-1\right)^{\ell_{2}} \mathrm{~d} \xi \mathrm{d} \omega .
\end{aligned}
$$

Let us check that by a suitable choice of $q$ one can ensure that the error term $\operatorname{Tr} Q_{q}(k)$ remains bounded as $k \rightarrow \infty$; this will certainly yield the desired asymptotics (1.16). Recall that by Lemma 2.5 , the operator $R_{q}(k)$ has an integral kernel in the class $C^{q}\left(\mathbb{S}^{d-1} \times \mathbb{S}^{d-1}\right)$ and its $C^{q}$-norm is $O\left(k^{-q}\right)$ as $k \rightarrow \infty$. Thus, by choosing $q$ sufficiently large, we can make sure that the estimate $\left(\|\cdot\|_{1}\right.$ is the trace norm)

$$
\left\|R_{q}(k)\right\|_{1}=O(1), \quad k \rightarrow \infty
$$

holds true. Next, using the estimates

$$
|\operatorname{Tr}(A B)| \leq\|A\|\|B\|_{1} \quad \text { and } \quad\|C\| \leq\|C\|_{1}
$$

and recalling that $Q_{q}(k)$ arose as a remainder term in the expansion of the brackets in the left hand side of (3.1), we obtain

$$
\left|\operatorname{Tr}\left(Q_{q}(k)\right)\right| \leq C\left(\ell_{1}, \ell_{2}\right) \max _{1 \leq j \leq \ell_{1}+\ell_{2}}\left\{\left\|R_{q}(k)\right\|_{1}^{j},\left\|\mathrm{Op}_{k^{-1}}[\sigma]\right\|^{\ell_{1}+\ell_{2}-j}\right\} .
$$

By (2.3), we have

$$
\left\|\mathrm{Op}_{k^{-1}}[\sigma]\right\|=O(1), \quad k \rightarrow \infty .
$$

Combining the last inequality with (3.3), we obtain that $\operatorname{Tr}\left(Q_{q}(k)\right)$ is bounded as $k \rightarrow \infty$, as required. 


\subsection{Application of the Weierstrass approximation theorem}

Lemma 3.2. Let $\ell_{0}$ be an even natural number and let $v, v_{k}(k \geq 1)$ be $\sigma$-finite measures on $\mathbb{T} \backslash\{1\}$ such that

$$
\int_{\mathbb{T}}|z-1|^{\ell_{0}} \mathrm{~d} v(z)<\infty, \quad \int_{\mathbb{T}}|z-1|^{\ell_{0}} \mathrm{~d} v_{k}(z)<\infty,
$$

for all $k$. Suppose that for all integers $\ell_{1} \geq 0, \ell_{2} \geq 0$ such that $\ell_{1}+\ell_{2} \geq \ell_{0}$, the relation

$$
\lim _{k \rightarrow \infty} \int_{\mathbb{T}}(z-1)^{\ell_{1}}(\bar{z}-1)^{\ell_{2}} d v_{k}(z)=\int_{\mathbb{T}}(z-1)^{\ell_{1}}(\bar{z}-1)^{\ell_{2}} d v(z)
$$

holds true. Then for any $\varphi \in C(\mathbb{T})$ such that $\varphi(z)|z-1|^{-\ell_{0}}$ is continuous on $\mathbb{T}$, the relation

$$
\lim _{k \rightarrow \infty} \int_{\mathbb{T}} \varphi(z) \mathrm{d} v_{k}(z)=\int_{\mathbb{T}} \varphi(z) \mathrm{d} v(z) .
$$

holds true.

Proof. Applying the Weierstrass approximation theorem to $\varphi(z)|z-1|^{-\ell_{0}}$, for any $\varepsilon>0$ we obtain a polynomial $\varphi_{0}(z)$ in $z, \bar{z}$ such that

$$
|\varphi(z)| z-\left.1\right|^{-\ell_{0}}-\varphi_{0}(z) \mid \leq \varepsilon, \quad z \in \mathbb{T} .
$$

Let us define $\varphi_{ \pm}(z)=\left(\operatorname{Re} \varphi_{0}(z) \pm \varepsilon\right)|z-1|^{\ell_{0}}$, then it follows from the above that

$$
\begin{gathered}
\varphi_{-}(z) \leq \operatorname{Re} \varphi(z) \leq \varphi_{+}(z), \quad z \in \mathbb{T}, \\
\varphi_{+}(z)-\varphi_{-}(z)=2 \varepsilon|z-1|^{\ell_{0}} .
\end{gathered}
$$

By the construction of $\varphi_{ \pm}$, it can be represented as a polynomial in $w=z-1$, $\bar{w}=\bar{z}-1$ involving only products $w^{\ell_{1}} \bar{w}^{\ell_{2}}$ with $\ell_{1}+\ell_{2} \geq \ell_{0}$. Thus, by (3.8) and (3.9) we can write

$$
\begin{gathered}
\int_{\mathbb{T}} \varphi_{-}(z) \mathrm{d} v(z) \leq \int_{\mathbb{T}} \operatorname{Re} \varphi(z) \mathrm{d} v(z) \leq \int_{\mathbb{T}} \varphi_{+}(z) \mathrm{d} v(z), \\
\int_{\mathbb{T}} \varphi_{-}(z) \mathrm{d} v_{k}(z) \leq \int_{\mathbb{T}} \operatorname{Re} \varphi(z) \mathrm{d} v_{k}(z) \leq \int_{\mathbb{T}} \varphi_{+}(z) \mathrm{d} v_{k}(z), \\
\int_{\mathbb{T}} \varphi_{+}(z) \mathrm{d} v(z)-\int_{\mathbb{T}} \varphi_{-}(z) \mathrm{d} v(z)=2 \varepsilon \int_{\mathbb{T}}|z-1|^{\ell_{0}} d v(z),
\end{gathered}
$$

where all integrals are absolutely convergent by (3.5). Now we can use (3.6) to pass to the limit in (3.11). Using (3.10) and (3.12) and denoting by $C$ the value of the 
integral in the right hand side of (3.12), we obtain

$$
\begin{aligned}
& \limsup _{k \rightarrow \infty} \int_{\mathbb{T}} \operatorname{Re} \varphi(z) \mathrm{d} v_{k}(z) \leq \int_{\mathbb{T}} \varphi_{+}(z) \mathrm{d} v(z) \leq \int_{\mathbb{T}} \operatorname{Re} \varphi(z) \mathrm{d} v(z)+2 \varepsilon C, \\
& \liminf _{k \rightarrow \infty} \int_{\mathbb{T}} \operatorname{Re} \varphi(z) \mathrm{d} v_{k}(z) \geq \int_{\mathbb{T}} \varphi_{-}(z) \mathrm{d} v(z) \geq \int_{\mathbb{T}} \operatorname{Re} \varphi(z) \mathrm{d} v(z)-2 \varepsilon C .
\end{aligned}
$$

Since $\varepsilon>0$ may be taken arbitrary small, this yields

$$
\lim _{k \rightarrow \infty} \int_{\mathbb{T}} \operatorname{Re} \varphi(z) \mathrm{d} v_{k}(z)=\int_{\mathbb{T}} \operatorname{Re} \varphi(z) \mathrm{d} v(z) .
$$

Since the same argument can be applied to the imaginary part of $\varphi$, we obtain the required statement.

3.3. Proof of Theorem 1.1. Recall that the measures $\mu_{k}$ and $\mu$ are defined in (1.5) and (1.6). By (1.7), (1.8), the conclusion of Lemma 3.1 can be written as

$$
\lim _{k \rightarrow \infty} k^{-d+1} \int_{\mathbb{T}}(z-1)^{\ell_{1}}(\bar{z}-1)^{\ell_{2}} d \mu_{k}(z)=\int_{\mathbb{T}}(z-1)^{\ell_{1}}(\bar{z}-1)^{\ell_{2}} d \mu(z) .
$$

Now it remains to apply Lemma 3.2 with $v_{k}=k^{-d+1} \mu_{k}$ and $v=\mu$.

Acknowledgements. The authors are grateful to N. Filonov and D. Yafaev for making valuable remarks on the preliminary version of the paper.

\section{References}

[1] S. Agmon, Some new results in spectral and scattering theory of differential operators on $\mathbb{R}^{n}$. Séminaire Goulaouic-Schwartz(1978/1979), Exp. No. 2. École Polytech., Palaiseau, 1979, 11 p. MR 0557513 Zbl 0406.35052

[2] Y. Aharonov and D. Bohm, Significance of electromagnetic potentials in the quantum theory. Phys. Rev. (2) 115 (1959), 485-491. MR 0110458 Zbl 0099.43102

[3] I. Alexandrova, Structure of the short range amplitude for general scattering relations. Asymptot. Anal. 50 (2006), 13-30. MR 2286935 Zbl 1135.35055

[4] M. Sh. Birman and D. R. Yafaev, Asymptotic behavior of the spectrum of the scattering matrix. J. Sov. Math. 25 (1984), 793-814. Original Russian: Zap. Nauchn. Sem. Leningrad. Otdel. Mat. Inst. Steklov. (LOMI) 110 (1981), 3-29. MR 0643971 (original Russian) Zbl Zbl 0501.35064 (original Russian) Zbl 0531.35062 (transl.)

[5] M. Sh. Birman and D. R. Yafaev, The scattering matrix for a perturbation of a periodic Schrödinger operator by decreasing potential. St. Petersburg Math. J. 6 (1995), 453-474. Original Russian: Algebra i Analiz 6 (1994), 17-39. MR 1301828 Zbl 0860.35088

[6] D. Bulger and A. Pushnitski, The spectral density of the scattering matrix for high energies. Comm. Math. Phys. 316 (2012), 693-704. MR 2993929 Zbl 1264.47015 
[7] K. Datchev, J. Gell-Redman, A. Hassell, and P. Humphries, Approximation and equidistribution of phase shifts: spherical symmetry. Preprint 2012. arXiv:1211.4959

[8] M. Dimassi and J. Sjöstrand, Spectral asymptotics in the semi-classical limit. London Mathematical Society Lecture Note Series 268. Cambridge University Press, Cambridge, 1999. MR 1735654 Zbl 0926.35002

[9] E. Doron and U. Smilansky, Semiclassical quantization of chaotic billiards: a scattering theory approach. Nonlinearity 5 (1992), 1055-1084. MR 1187738 Zbl 0770.58043

[10] Yu. V. Egorov, A. I. Komech, and M. A. Shubin, Elements of the modern theory of partial differential equations. Translated from the 1988 Russian original by P. C. Sinha. Reprint of the original English edition from the series Encyclopaedia of Mathematical Sciences (Partial differential equations II, Encyclopaedia Math. Sci. 31, Springer, Berlin, 1994). Springer, Berlin, 1999. MR 1693807 Zbl 0946.35001

[11] L. S. Koplienko, The spectrum of the scattering matrix. Izv. Vyš̌. Učebn. Zaved. Matematika 1971 (108), 54-61. In Russian. MR 0290176

[12] A. Pushnitski, G. Raikov, and C. Villegas-Blas, Asymptotic density of eigenvalue clusters for the perturbed Landau Hamiltonian. Comm. Math. Phys. 320 (2013), 425-453. MR 3053767 Zbl 06176793

[13] S. N. M. Ruijsenaars, The Aharonov-Bohm effect and scattering theory. Ann. Physics 146 (1983), 1-34. MR 0701261 Zbl 0554.47003

[14] M. A. Shubin, Pseudodifferential operators and spectral theory. Translated from the Russian by S. I. Andersson. Springer Verlag, Berlin etc., 1987. Original Russian: Nauka, Moskow, 1978. Second English ed.: Springer Verlag, Berlin, 2001. MR 0509034 (original Russian) MR 0883081 (transl.) MR 1852334 (second ed.) Zbl 0451.47064 (original Russian) Zbl 0616.47040 (transl.) Zbl 0980.35180 (second ed.)

[15] M. E. Taylor, Pseudodifferential operators. Princeton Mathematical Series 34. Princeton University Press, Princeton, N.J., 1981. MR 0618463 Zbl 0453.47026

[16] L. Thomas, C. Villegas-Blas, Asymptotics of Rydberg states for the hydrogen atom. Comm. Math. Phys. 187 (1997), 623-645. MR 1468317 Zbl 0998.47050

[17] A. Uribe, C. Villegas-Blas, Asymptotics of spectral clusters for a perturbation of the hydrogen atom. Comm. Math. Phys. 280 (2008), 123-144. MR 2391192 Zbl 1145.81052

[18] A. Weinstein, Asymptotics of eigenvalue clusters for the Laplacian plus a potential. Duke Math. J. 44 (1977), 883-892. MR 0482878 Zbl 0385.58013

[19] D. Yafaev, High energy and smoothness asymptotic expansion of the scattering amplitude. J. Functional Analysis 202 (2003), 526-570. MR 1990537 Zbl 1045.35059

[20] D. Yafaev, Scattering by magnetic fields. St. Petersburg Math. J. 17 (2006), 875-895. Original Russian: Algebra i Analiz 17 (2005), 244-272. MR 2241430 Zbl 1110.47065

[21] D. Yafaev, Mathematical scattering theory. Analytic theory. Mathematical Surveys and Monographs 158. Amer. Math. Soc., Providence, R.I, 2010. MR 2598115 Zbl 1197.35006

[22] S. Zelditch and M. Zworski, Spacing between phase shifts in a simple scattering problem. Comm. Math. Phys. 204 (1999), 709-729. MR 1707603 Zbl 0946.47006 
Received September 1, 2012; revised November 2, 2012

Daniel Bulger, Department of Mathematics, King's College London, Strand, London, WC2R 2LS, U.K.

E-mail: daniel.bulger@kcl.ac.uk

Alexander Pushnitski, Department of Mathematics, King's College London, Strand, London, WC2R 2LS, U.K.

E-mail: alexander.pushnitski@kcl.ac.uk 